INRA Prod. Anim.,

2011, 24 (3), 235-244

\title{
Analyse de la mortalité bovine en France de 2003 à 2009
}

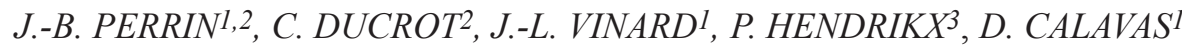 \\ ${ }^{1}$ Agence nationale de sécurité sanitaire (Anses), Laboratoire de Lyon, Unité Epidémiologie, \\ 31, avenue Tony Garnier, F-69364 Lyon Cedex 07, France \\ 2 INRA, UR346 Unité d'épidémiologie animale, F-63122, Saint-Genès-Champanelle, France \\ 3 Agence nationale de sécurité sanitaire (Anses), Direction scientifique des laboratoires, \\ 27-31 avenue du général Leclerc F-94701 Maisons-Alfort Cedex, France
}

Courriel : jean-baptiste.perrin@anses.fr

La Base de Données Nationale d'Identification bovine (BDNI) permet de connaître précisément et à tout instant la composition de la population bovine française (soit 20 millions d'individus en moyenne) et la mortalité qui y est associée. Cet article présente l'analyse des taux de mortalité dans le cheptel bovin français et leur évolution depuis 2003, en fonction du sexe, de l'âge et du type de production des animaux. Cette étude apporte des données de référence utilisables pour évaluer des situations observées en élevage.

La mortalité chez les bovins (hors abattage) est un problème économique, en raison des pertes directes (valeur de la carcasse, production, génétique) et indirectes (coût du remplacement et surcharge de travail) induites pour les éleveurs (Holleville et Michenot 2009). C'est également un problème éthique, qui pose la question du bien-être des animaux d'élevage. La mortalité constitue aussi un indicateur général de la santé du cheptel qui peut jouer un rôle important dans la surveillance épidémiologique (Perrin et al 2010). Pourtant, malgré son importance, rares sont les études consacrées à ce sujet, alors que de nombreuses données sont désormais disponibles.

En effet, depuis le règlement européen $N^{\circ} 1760 / 2000$, chaque état membre est tenu d'administrer une base de données nationale sur l'identification des bovins. Ces bases enregistrent tous les mouvements d'animaux (dont les naissances et les morts) vers et depuis les exploitations bovines. En France, la Base de Données Nationale d'Identification bovine (BDNI) centralise l'ensemble de ces notifications, ce qui permet de connaître précisément et à tout instant la composition de la population bovine (soit 20 millions d'individus en moyenne) et la mortalité qui y est associée.

L'analyse de ces notifications permet, via des indicateurs appropriés, d'évaluer l'importance et l'évolution de la mortalité dans le cheptel bovin depuis 2003. La Direction Générale de l'Alimentation
(DGAL), l'Agence nationale de sécurité sanitaire de l'alimentation, de l'environnement et du travail (Anses) et l'Institut National de la Recherche Agronomique (INRA), se sont rassemblés dans le cadre du projet OMAR (Observatoire de la Mortalité des Animaux de Rente) pour exploiter et valoriser les données collectées.

L'objectif de cet article est de présenter des valeurs de référence de la mortalité bovine en France et de son évolution entre 2003 et 2009, en considérant différents indicateurs (effectifs, taux et risques de mortalité) et différents facteurs explicatifs (âge, sexe, type de production...).

\section{1 / Matériel et méthode}

\section{1 / Collecte des données}

Les notifications d'identification et de mouvements des bovins sont transmises par les éleveurs aux Établissements Départementaux de l'Elevage (EDE), puis centralisées dans la BDNI après un contrôle de conformité. De 2003 à 2009 , la base a enregistré près de 155 millions de notifications de mouvements (indiquant le numéro d'exploitation, la date et le type de mouvement (entrée ou sortie), la cause d'entrée (naissance ou achat) ou de sortie (vente, abattage, équarrissage) effectués par 75 millions de bovins (pour lesquels ont été notamment enregistrés le numéro national, la date de naissance, le sexe et la race). En théorie cette base est exhaustive car les détenteurs des animaux sont réglementairement tenus de notifier ces informations, et font à ce titre l'objet de contrôles réguliers par les services vétérinaires départementaux.

La base de données a été nettoyée, les données aberrantes ont été corrigées lorsque l'anomalie était facilement identifiable (sortie pour cause «boucherie» suivie d'une entrée dans un autre élevage), et supprimées dans le cas contraire. Les départements d'Outremer, de la Corse ainsi que ceux de Paris petite couronne ont été exclus de l'analyse en raison de la quantité et de la fiabilité moindres des données dans ces zones.

\section{2 / Traitement des données}

Le nombre de morts de bovins a directement été déduit du nombre de notifications de sorties pour cause «mortalité» transmises par les éleveurs aux EDE. Les effectifs bruts de mortalité ayant peu d'intérêt s'ils ne sont pas rapportés à la population correspondante, un algorithme a été développé afin de calculer le dénominateur (population bovine vivante) nécessaire à l'estimation des taux et risques de mortalité. Pour une période donnée, l'algorithme calcule le nombre de jours de participation de chaque animal au groupe populationnel étudié, puis somme les résultats pour obtenir le nombre total de bovin-jours correspondant à ce groupe. Chaque mouvement individuel survenant au 
Figure 1. Principe de calcul de l'algorithme population. Exemple du nombre de bovin-jours au cours d'une semaine pour un groupe d'âge $n$, avec la participation de 5 individus types ( $A$ à $E$ ).

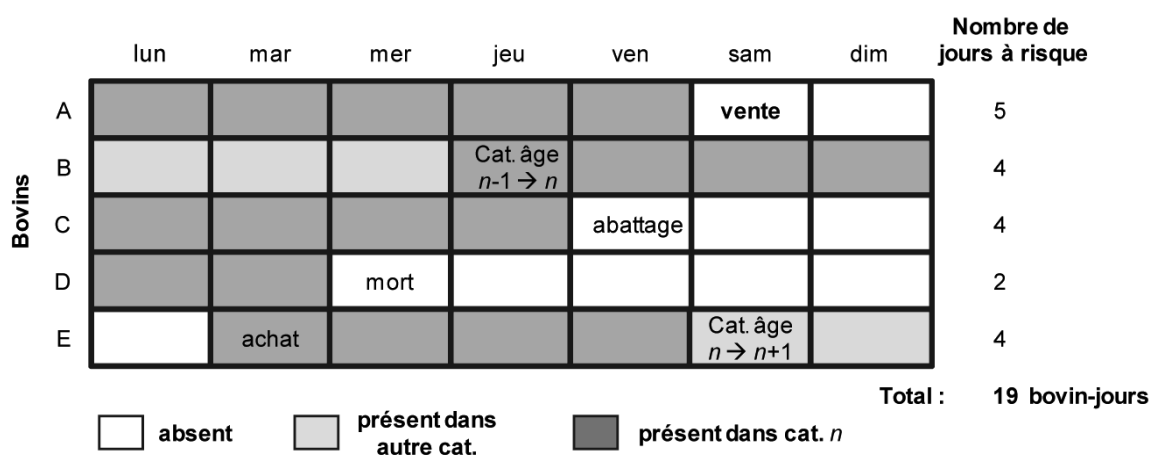

cours de cette période, qu'il s'agisse d'une entrée (naissance, achat) ou d'une sortie (vente, abattage, équarrissage) est pris en compte à sa date de réalisation. Par ailleurs, si le sous-groupe populationnel est défini par l'âge, l'algorithme prend en compte les dates de changement de catégorie d'âge (déterminées à partir de la date de naissance de l'animal) pour chaque individu.

Par exemple, l'algorithme permet de calculer le nombre de bovin-jours correspondant au groupe «vaches laitières de deux à cinq ans» en France au cours d'une semaine déterminée (figure 1). Le calcul de ces temps de présence cumulés donne accès à un dénominateur précis permettant de calculer sans approximation des taux d'incidence de mortalité (définition ci-après), quels que soient l'échelle spatio-temporelle et le sous-groupe populationnel considérés.

Les animaux nés et morts le même jour sont comptabilisés dans le dénominateur comme un bovin-jour.

\section{3 / Estimation des indicateurs de mortalité}

Nous utilisons pour décrire la mortalité dans cet article deux indicateurs dont l'interprétation est différente : le taux et le risque de mortalité.

\section{a) Taux de mortalité}

Le taux d'incidence de mortalité, ciaprès simplement dénommé taux de mortalité, est assimilable à une vitesse et s'exprime en nombre de morts par individu-jours. Par exemple dans cet article, les taux de mortalité sont exprimés en nombre de morts par 100000 bovin-jours : leur valeur correspond au nombre de morts que l'on observerait chaque jour dans une population constante de 100000 individus. Le taux de mortalité $(\lambda)$ se calcule de la manière suivante (Jougla 1997) :

$$
\lambda_{(t)}=\frac{m_{[t, t+\Delta t]}}{B J_{[t, t+\Delta t}}
$$

Avec respectivement $\mathrm{m}_{[\mathrm{t}, \mathrm{t}+\Delta \mathrm{t}]}$ le nombre de morts survenant au cours de la période $[\mathrm{t}, \mathrm{t}+\Delta \mathrm{t}]$ dans la population considérée, et $\mathrm{BJ}_{[\mathrm{t}, \mathrm{t}+\Delta \mathrm{t}]}$ le nombre de bovin-jours à risque (c'est à dire vivants) constituant cette population.

Même si les caractéristiques d'une population sont fixées, le taux de mortalité doit être considéré comme aléatoire; aussi les estimations des taux de mortalité sont accompagnées d'intervalles de confiance (Jougla 1997):

$$
T=\lambda \pm z_{\alpha / 2} \sqrt{\frac{\lambda}{B J}}
$$

avec $\mathrm{T}$ le taux de mortalité théorique, $\lambda$ le taux de mortalité observé, BJ le nombre de bovin-jours correspondant, et $Z_{\alpha / 2}$ la valeur de la loi normale centrée - réduite $Z$ telle que $\mathrm{P}\left(\mathrm{Z}>\mathrm{Z}_{\alpha / 2}\right)$ $=\alpha$.

Des taux de mortalité spécifiques ont été calculés pour différents sous-groupes populationnels, définis selon l'âge, le sexe et le type de production (laitier ou allaitant) des animaux. Un type de production a été attribué à chaque animal selon sa race (la liste du type de production en fonction de la race a été constituée à dire d'experts). Les dix catégories d'âge ont quant à elles été définies sur des critères zootechniques afin d'être représentatives de certaines étapes de la carrière d'un bovin.

\section{b) Risque de mortalité}

Connaissant le taux de mortalité (considéré comme constant) qui s'exerce sur une population, on peut calculer la probabilité qu'a un animal de mourir dans un intervalle de temps déterminé (t). Cette probabilité, correspondant au risque de mortalité $\mathrm{R}_{(\mathrm{t})}$, dépend de l'intervalle de temps choisi :

$$
R_{(t)}=1-\exp (-\lambda \times t)
$$

Ainsi, dans une population soumise à un taux de mortalité $\lambda$ de 10 pour 100000 bovin-jours, la probabilité pour un bovin de mourir au bout de 10 jours $\left(\mathrm{R}_{10}\right)$ sera de $0,1 \%$, celle de mourir au bout de 100 jours $\left(\mathrm{R}_{100}\right)$ sera de $1 \%$, et celle de mourir au bout de 365 jours $\left(R_{365}\right)$ sera de $3,6 \%$.

Nous avons estimé des taux de mortalité par catégorie d'âge, puis les avons convertis en risques de mortalité, dont la signification est différente pour les catégories supérieures et inférieures à un an.

Pour les catégories d'âge inférieures à un an, le risque estimé correspond à la probabilité qu'a un animal de mourir dans cette catégorie d'âge sachant qu'il l'a atteinte : $t$ correspond à la durée de la catégorie d'âge (sept jours pour les veaux de moins de sept jours, 24 pour les animaux de sept jours à un mois, 31 pour les animaux de un à deux mois, etc.).

Pour les catégories d'âge supérieures à un an, le risque calculé correspond à la probabilité qu'a un animal de mourir dans cette catégorie d'âge dans l'année sachant qu'il l'a atteinte : $t$ correspond à 365 jours.

Ainsi, la forme de cet indicateur (pourcentage) est plus familière que celle du taux de mortalité, mais son interprétation est en revanche plus délicate.

\section{4 / Modélisation des séries hebdomadaires}

La saisonnalité et la tendance des taux de mortalité ont été décrites à l'aide de modèles de Poisson avec sur dispersion, qui ont été ajustés sur les séries hebdomadaires de mortalité. Pour chaque population, l'évolution temporelle des taux a été modélisée par une tendance linéaire et un facteur saisonnier mensuel :

$$
\begin{gathered}
m \sim \text { Poisson }(\mu) \quad V(m)=\varphi \mu \\
\log \left(\mu_{s}\right)=\log \left(B J_{s}\right)+\beta_{0}+\beta_{1} \times t+\sum_{i=f e v}^{\text {dec }}\left[\beta_{i} X_{i}\right]
\end{gathered}
$$

avec $\mathrm{m}$ le nombre de morts, $\mu$ et $\mathrm{V}(\mathrm{m})$ respectivement l'espérance et la variance de $\mathrm{m}, \varphi$ le paramètre de surdispersion, $\mathrm{s}$ le temps (de 1 à 366, soit le nombre de semaines de 2003 à 2009), i le mois de la semaine $\mathrm{t}, \mathrm{X}_{\mathrm{i}}$ une variable indicatrice valant 1 si la semaine considérée appartient au mois $i$ et 0 sinon, et $\beta_{0}$ l'ordonnée à l'origine, $\beta_{1}$ la tendance et $\beta_{j}$ les 11 paramètres saisonniers (le mois de janvier étant le mois de référence). 
Figure 2. Taux de mortalité par catégorie d'âge et type de production (laitier en rouge et allaitant en noir) des bovins en France pour la période 2003-2009.

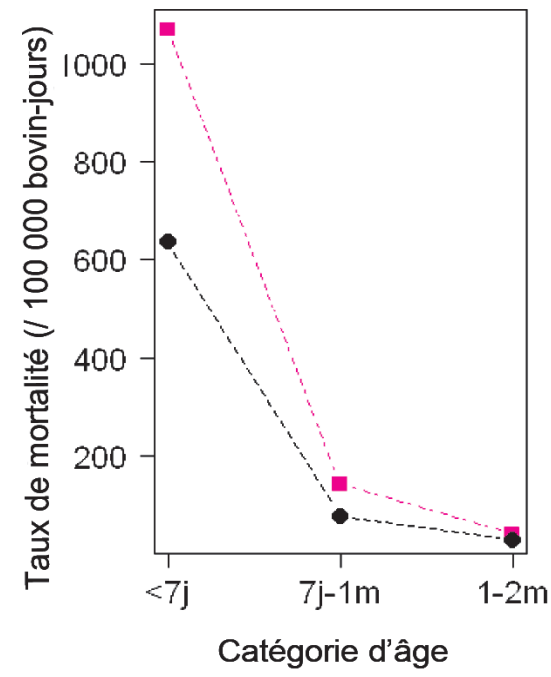

\section{2 / Résultats et discussion}

De 2003 à 2009, 8807067 morts de bovins ont été notifiées par les éleveurs en France métropolitaine, hors région parisienne.

Les valeurs des taux de mortalité estimés sont représentées par catégorie d'âge et type de production sur la figure 2. Dans les deux types de production, l'évolution des taux de mortalité en fonction de l'âge est globalement similaire : les taux de mortalité sont au plus haut chez les veaux de moins de sept jours (catégorie qui prend en compte les veaux mort-nés) et décroissent fortement jusqu'à atteindre un minimum entre un et deux ans, puis croissent à nouveau.

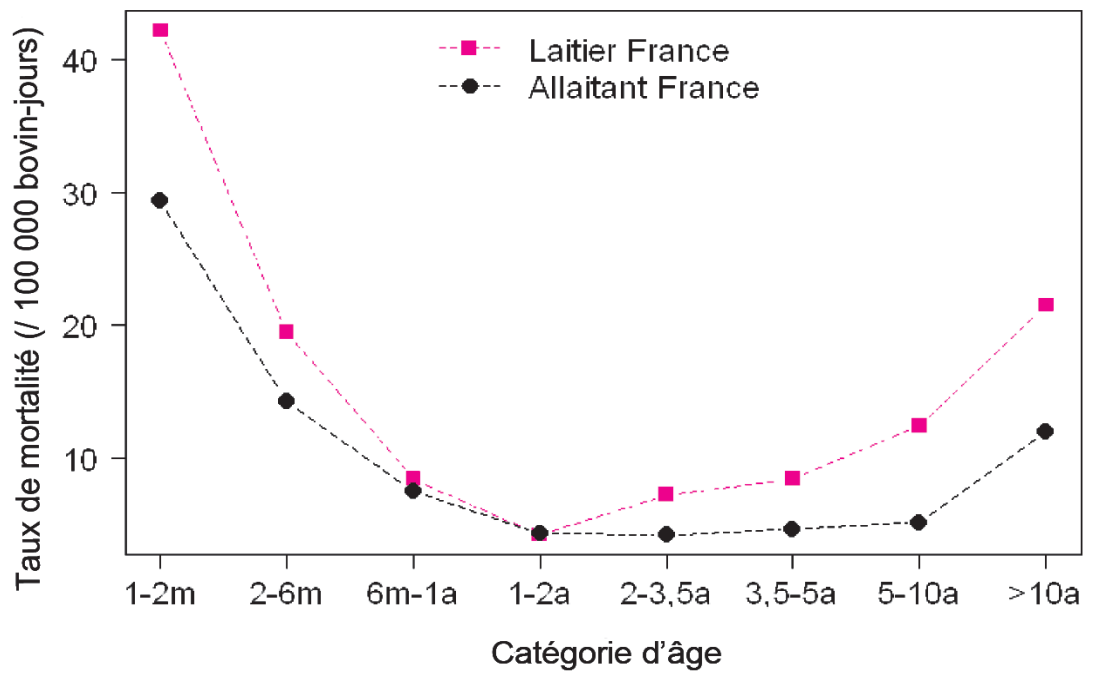

Le nombre de bovin-jours et de morts de 2003 à 2009, ainsi que les taux et risques de mortalité correspondants sont indiqués par catégories d'âge dans le tableau 1 pour les bovins allaitants, et dans le tableau 2 pour les bovins laitiers. Les taux de mortalité observés chez les bovins laitiers sont significativement plus élevés que chez les allaitants pour toutes les catégories d'âge sauf celle de un à deux ans, où le taux est supérieur chez les bovins allaitants.

\section{1 / Mortalité des veaux}

Près de $70 \%$ des notifications enregistrées de 2003 à 2009 correspondaient à des morts d'animaux de moins de six mois. La distribution du nombre de veaux morts en fonction du sexe, du type de production et de l'âge à la mort est représentée sur la figure 3 .

\section{a) Mortalité périnatale}

Sur les 55727233 naissances notifiées entre 2003 et 2009 , la proportion de veaux morts avant $48 \mathrm{~h}$, considérés à ce titre comme veaux mort-nés (VMN), était de 5,2\%. Cette proportion, que nous avons assimilé au risque de mortalité périnatale, était plus importante chez les bovins laitiers (7\%) que chez les bovins allaitants (4\%), et plus importante chez les mâles que chez les femelles, tant chez les bovins laitiers $(7,9 \%$ et $6,0 \%$ respectivement pour les mâles et les femelles) que chez les bovins allaitants $(4,7 \%$ et $3,2 \%)$.

Dans la littérature, la mortalité périnatale est définie comme la mortalité de veaux à terme et jusqu'à 24 ou 48 heures, selon les auteurs. Les valeurs que nous avons obtenues sont comprises

Tableau 1. Cheptel allaitant - Population bovine de 2003 à 2009, nombre de morts, taux et risque de mortalité par catégorie d'âge.

\begin{tabular}{|c|c|c|c|c|c|c|}
\hline $\begin{array}{c}\text { Catégorie } \\
\text { d'âge }\end{array}$ & $\begin{array}{c}\text { Nb. de bovin- } \\
\text { jours (03-09) }\end{array}$ & $\begin{array}{c}\text { Nb de morts } \\
\text { (03-09) }\end{array}$ & $\begin{array}{c}\text { Nb d'animaux } \\
\text { vivants moyen* }\end{array}$ & $\begin{array}{c}\text { Nb de morts } \\
\text { moyen* }\end{array}$ & Taux ** $^{*}$ Risque*** $^{*}$ \\
\hline$<7 j$ & 261776856 & 1666017 & 102177 & 650 & 636 & 4,36 \\
\hline $7 j-1 m$ & 712100796 & 555793 & 277947 & 217 & 78 & 1,63 \\
\hline $1-2 m$ & 918385754 & 269999 & 358464 & 105 & 29,4 & 0,88 \\
\hline $2-6 m$ & 3499993204 & 496883 & 1366118 & 194 & 14,2 & 1,69 \\
\hline $6 m-1 a$ & 3946360305 & 294723 & 1540344 & 115 & 7,5 & 1,35 \\
\hline $1-2 a$ & 4914987596 & 212356 & 1918418 & 83 & 4,3 & 1,56 \\
\hline $2-3,5 a$ & 4533457598 & 188293 & 1769499 & 73 & 4,2 & 1,5 \\
\hline $3,5-5 a$ & 2811004356 & 129842 & 1097191 & 51 & 4,6 & 1,67 \\
\hline $5-10 a$ & 5289471650 & 268947 & 2064587 & 105 & 5,1 & 1,84 \\
\hline$>10 a$ & 1744946994 & 209085 & 681088 & 82 & 12 & 4,28 \\
\hline TOTAL & 28632485109 & 4291938 & 11175833 & 1675 & 15 & - \\
\hline
\end{tabular}

* par jour ** par 100000 bovin-jours *** Risque moyen annuel en \%. 
Tableau 2. Cheptel laitier - Population bovine de 2003 à 2009, nombre de morts, taux et risque de mortalité par catégorie d'âge.

\begin{tabular}{|c|c|c|c|c|c|c|}
\hline $\begin{array}{c}\text { Catégorie } \\
\text { d'âge }\end{array}$ & $\begin{array}{c}\text { Nb. de bovin- } \\
\text { jours (03-09) }\end{array}$ & $\begin{array}{c}\text { Nb de morts } \\
(\mathbf{0 3 - 0 9 )}\end{array}$ & $\begin{array}{c}\text { Nb d'animaux } \\
\text { vivants moyen* }\end{array}$ & $\begin{array}{c}\text { Nb de morts } \\
\text { moyen* }^{*}\end{array}$ & Taux $^{* *}$ & Risque $^{* * *}$ \\
\hline$<7 j$ & 164493548 & 1758164 & 64205 & 686 & 1069 & 7,21 \\
\hline $7 \mathrm{j}-1 \mathrm{~m}$ & 417600943 & 597929 & 162998 & 233 & 143,2 & 2,96 \\
\hline $1-2 \mathrm{~m}$ & 549111601 & 231931 & 214329 & 91 & 42,2 & 1,26 \\
\hline $2-6 \mathrm{~m}$ & 2074813457 & 404644 & 809841 & 158 & 19,5 & 2,31 \\
\hline $6 \mathrm{~m}-1 \mathrm{a}$ & 2085487067 & 175269 & 814007 & 68 & 8,4 & 1,52 \\
\hline $1-2 a$ & 3893649859 & 164157 & 1519770 & 64 & 4,2 & 1,53 \\
\hline $2-3,5 a$ & 4590973757 & 331436 & 1791949 & 129 & 7,2 & 2,6 \\
\hline $3,5-5 a$ & 3294876453 & 276300 & 1286056 & 108 & 8,4 & 3,01 \\
\hline $5-10 a$ & 4220223182 & 522789 & 1647238 & 204 & 12,4 & 4,42 \\
\hline$>10 a$ & 358555284 & 77002 & 139951 & 30 & 21,5 & 7,54 \\
\hline TOTAL & 21649785151 & 4539621 & 8450344 & 1771 & 21 & - \\
\hline
\end{tabular}

* par jour ${ }^{* *}$ par 100000 bovin-jours *** Risque moyen annuel en $\%$.

dans l'intervalle des valeurs de mortalité périnatale proposées.

Une revue des études menées sur la mortalité périnatale des veaux laitiers rapporte par exemple des taux variant de $2 \%$ à $10 \%$ selon les pays (Mee et al 2008). Dans des cheptels allaitants d'Amérique du Nord, une proportion de veaux mort-nés de $2,6 \%$ (Waldner et al 2010), voire $2,9 \%$ (USDA 2010) et un risque de mortalité de $4,4 \%$ dans les 24 heures (Ganaba et al 1995) ont été rapportés. Des risques de mortalité dans les 72 heures de $4,9 \%$ (5,6 et $4,0 \%$ pour les mâles et les femelles respective- ment) et de $9,7 \%$ ont été observés respectivement en Espagne (Goyache et al 2003) et en France dans la région Bretagne (Offredo 2010).

La mortalité périnatale est associée à de multiples facteurs de risque tels que les caractéristiques du père, le poids, le sexe et la présentation du veau à la naissance, la gémellité, la durée de la gestation, la saison, l'âge, l'existence de maladies ou encore la race, le degré de parité et la taille du pelvis de la mère (Bellows et al 1987, Bleul 2011, Mee et al 2008). Nous avons identifié une différence importante entre les cheptels laitiers et allaitants qui s'explique aisément puisque le type de production (que nous avons déterminé à partir de la race) est associé à un certain nombre de ces facteurs : poids à la naissance, durée de gestation, taille du pelvis de la mère, saison de vêlage, âge au premier vêlage, etc. La mortalité périnatale est traditionnellement associée aux vêlages difficiles ou dystociques, mais certaines études suggèrent qu'une part croissante de ces morts surviennent après un vêlage ayant nécessité peu ou pas d'assistance (Mee et al 2008).

L'écart que nous avons observé entre les sexes a déjà été rapporté par d'autres

Figure 3. Diagrammes du nombre de bovins morts en France de 2003 à 2009, par type de production (laitiers en haut, allaitants en bas), sexe et âge (en jours). Gauche : Veaux Mort-Nés (VMN), Droite : veaux de moins de 6 mois.

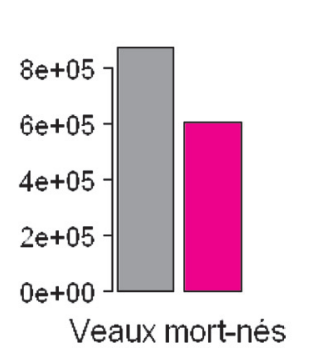

$$
\left.\begin{array}{r}
60000 \\
50000- \\
40000- \\
30000- \\
20000- \\
10000- \\
0
\end{array}\right]
$$

$\mathrm{Nb}$. cle morts

\section{Laitiers}
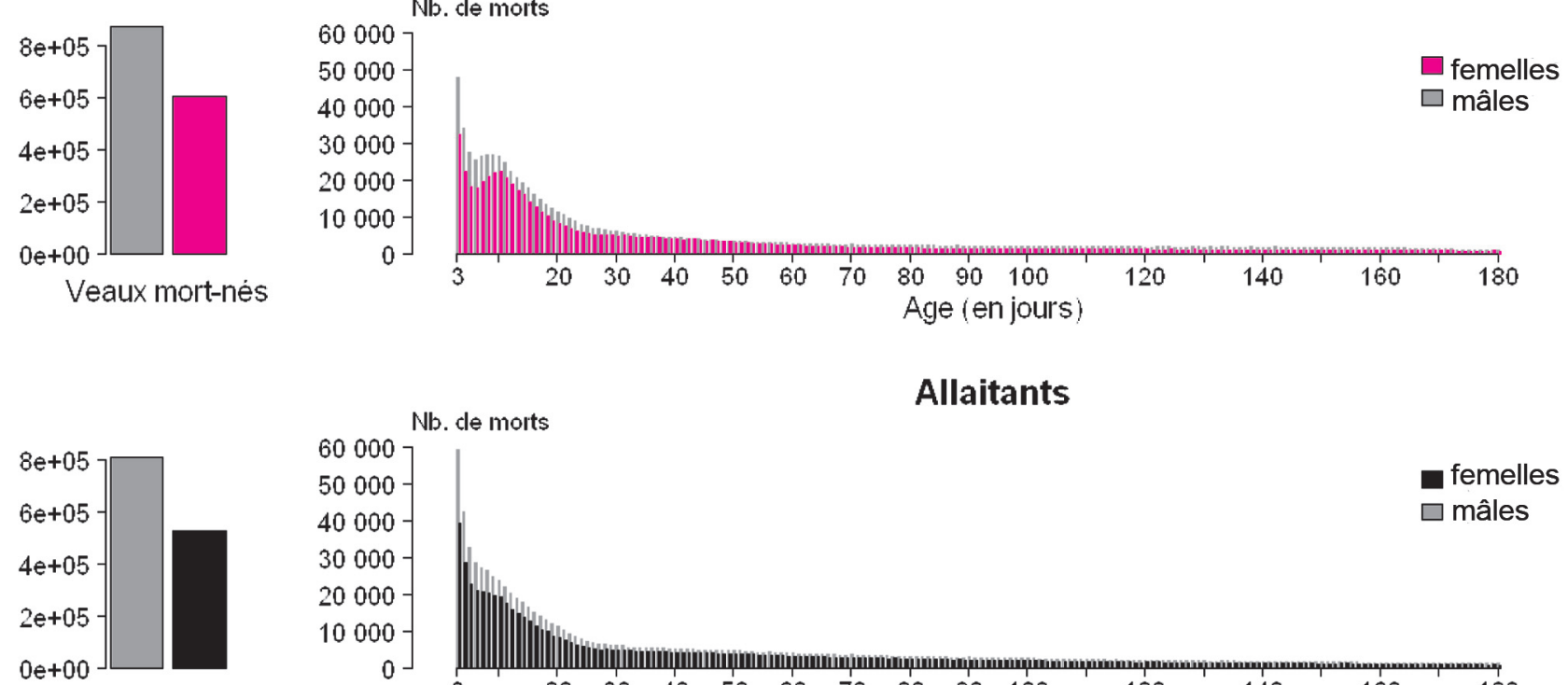

Veaux mort-nés
$\mathrm{Nb}$. cle morts

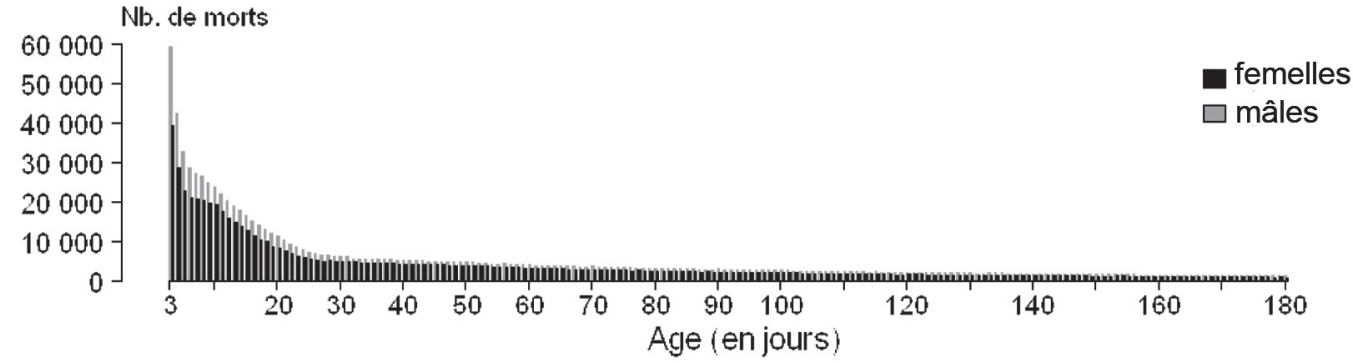


auteurs (Bleul 2011, Johanson et Berger 2003, Lombard et al 2007, Martinez et al 1983, Nix et al 1998), mais ce constat n'est pas systématique (Rao et Nagarcenkar 1980). Il est généralement attribué à un poids à la naissance supérieur chez les mâles (Bleul 2011) qui augmente le taux de dystocies. Un effet persistant du sexe sur la mortalité périnatale a cependant été mis en évidence après prise en compte du risque de dystocie (Mee et al 2008). Ainsi cette différence pourrait aussi être due à une moins bonne viabilité des mâles, comme cela est le cas dans l'espèce humaine (Drevenstedt et al 2008). Nous avons d'ailleurs observé, comme chez l'Homme, un sex-ratio à la naissance en faveur des mâles de 1,07 (une valeur identique à celle récemment estimée dans le cheptel bovin suisse (Bleul 2011)).

\section{b) Mortalité postnatale}

Après trois jours, le nombre de notifications enregistrées dans la BDNI ne décroit pas de façon exponentielle en fonction de l'âge à la mort : on observe à dix jours un pic chez les laitiers et une inflexion chez les allaitants (figure 3). Cette courbe atypique pourrait être un artefact dû au système de notification de la mortalité des veaux en France. En effet, l'identification des veaux morts en-dessous de sept jours (âge où celle-ci est strictement obligatoire) n'est pas exigée dans tous les départements. Une partie des veaux morts entre trois et sept jours a donc pu être en fait déclarée comme des veaux mort-nés, ce qui expliquerait le déficit apparent de morts au cours de cette période. Si tel était le cas, les risques de mortalité avant 48 heures, décrits dans le paragraphe précédent pourraient avoir été surestimés.

Ce biais n'affecte pas en revanche le risque de mortalité à sept jours présenté dans les tableaux 1 et 2 , puisque celui-ci prend à la fois en compte les veaux mortnés et les veaux déclarés morts lors de la première semaine. Celui-ci est plus élevé chez les veaux laitiers $(7,2 \%)$ que chez les veaux allaitants $(4,4 \%)$. En revanche, si l'on omet les VMN, le risque de mortalité de deux à sept jours est plus élevé chez les bovins allaitants $(0,38 \%)$ que chez les bovins laitiers $(0,23 \%)$. Une étude menée en Suisse a observé un phénomène similaire et attribue cette différence à la meilleure protection immunitaire, dont bénéficieraient les veaux laitiers car leur prise de colostrum est mieux contrôlée par les éleveurs (Bleul 2011). Pour le cheptel français ces résultats doivent toutefois être considérés avec précaution, considérant le biais probable dans la notification des veaux mort-nés évoqué ci-dessus.
Pour les animaux ayant atteint sept jours, le risque de mourir avant un, deux et six mois était respectivement de $1,6 \%, 2,5 \%$ et $4,1 \%$ chez les allaitants, et de $3,0 \%, 4,2 \%, 6,4 \%$ chez les laitiers. Ces valeurs semblent globalement compatibles avec les risques de mortalité postnatale proposées dans la littérature, mais la comparaison est difficile car les études portent sur des catégories d'âges très variables.

Par exemple dans le cheptel laitier, plusieurs valeurs ont été proposées pour le risque de mortalité de un jour à trois mois : 3,1\% (Svensson et al 2006) et 9\% (Torsein et al 2011) en Suède, 4,2\% et $13,8 \%$ au Danemark (Torsein et al 2011), 5,6\% (Virtala et al 1996) et 9,4\% (Losinger et Heinrichs 1997) aux EtatsUnis. Des risques de un jour à six mois variant du simple au double selon la race ont par ailleurs été estimés au Danemark: 6,6\% chez des veaux de race Holstein (Hansen et al 2003) et $12,6 \%$ chez des veaux de race Jersey (Norberg 2008). D'autres auteurs rapportent que $7,8 \%$ des veaux (dont $4,1 \%$ de veaux mort-nés) mouraient au cours de la première année de vie en Norvège (Gulliksen et al 2009), ou que les veaux d'une race mixte avaient un risque de mourir de $1,9 \%$ de $48 \mathrm{~h}$ à un mois, de $1,4 \%$ de un à six mois et de $5,6 \%$ de la naissance au premier vêlage en Autriche (Fuerst-Waltl et Fuerst 2010).

Dans le cheptel allaitant, les valeurs proposées pour le risque de mortalité sont tout aussi variables : $7,7 \%$ pour des animaux de un jour à un mois (Ganaba et al 1995), 4,5\% (Wittum et al 1993 ) et $6,4 \%$ (USDA 2010) de la naissance au sevrage, $3,7 \%$ de $72 \mathrm{~h}$ au sevrage (Goyache et al 2003), 2,1\% de quatre jours au sevrage (Patterson et al 1987), 6,4\% la première semaine puis $2,8 \%$ de une semaine au sevrage (Azzam et al 1993), 4,8\% de morts (par diarrhée uniquement) dans les dix jours suivant la naissance (Schumann et al 1990).

Après la naissance et jusqu'à six mois, les taux de mortalité que nous avons estimés étaient beaucoup plus élevés pour les veaux laitiers que pour les veaux allaitants. En dehors des dystocies, qui sont associées à une surmortalité jusqu'à l'âge de un (Lombard et al 2007) voire de quatre mois (Bleul 2011), les maladies respiratoires et les diarrhées sont fréquemment rapportées comme principales causes de mortalité postnatale (Gulliksen et al 2009, Lombard et al 2007, Svensson et al 2006). La mortalité plus importante chez les veaux laitiers pourrait être liée à la manière dont ils sont élevés, avec un sevrage et un allotement plus précoces augmentant le risque de maladies.
On constate globalement que les animaux ayant une valeur économique moindre (veaux laitiers mâles par rapport aux femelles, veaux laitiers par rapport aux veaux allaitants) présentent des taux de mortalité péri et post-nataux supérieurs, suggérant qu'ils font peutêtre l'objet de moins de soins vétérinaires lorsque leur état de santé se dégrade.

\section{2 / Mortalité des jeunes bovins}

Chez les bovins allaitants, le risque de mortalité était de $1,3 \%$ entre 6 mois et 1 an, et de 2,9\% entre 6 mois et 2 ans. Ces risques étaient respectivement de $1,5 \%$ et $3 \%$ chez les laitiers. De même, les valeurs du risque de mortalité des jeunes bovins dans la littérature sont faibles : $1 \%$ de six mois au premier vêlage (Fuerst-Waltl et Fuerst 2010), 2,2\% entre sept mois et le premier vêlage (Svensson et al 2006).

Les bovins de six mois à deux ans représentaient la catégorie d'âge pour laquelle le taux de mortalité est le plus bas et pour laquelle la différence entre les types de production est la plus réduite, probablement parce qu'à ces âges, les conditions d'élevage des animaux diffèrent peu selon le type de production, et les exposent à des risques de mortalité limités.

\section{3 / Mortalité des adultes}

Au delà de six mois, la proportion de mâles parmi les bovins morts chute rapidement en raison de leur élimination de la population vivante, par abattage ou exportation et leur proportion est négligeable au-delà de deux ans. En revanche, l'évolution du nombre de femelles mortes est très variable selon le type de production et la race (figure 4).

Un pic de mortalité est visible à trois ans dans les deux types de production, mais chez les races laitières l'augmentation est plus précoce (dès deux ans chez les Holstein). Des pics secondaires sont observables à intervalles réguliers (d'environ un an) après le pic principal chez les races allaitantes et de manière plus limitée chez les races laitières. Ces distributions confirment l'importance de la surmortalité associée à la période peripartum : les pics observés surviennent aux âges de vêlage standards des vaches en production. L'augmentation du nombre de morts débute plus tôt chez les laitières certainement en raison de la pratique du vêlage précoce, plus répandue dans ce type de production. Le crénelage est ensuite beaucoup plus marqué dans les distributions relatives aux races allaitantes, ce qui pourrait indiquer que l'âge au premier vêlage puis l'intervalle vêlage-vêlage (environ un an) est plus régulier chez ces dernières 
Figure 4. Diagrammes du nombre de bovins morts au delà de six mois en France de 2003 à 2009, en fonction du sexe et de la race (laitiers à gauche : femelles en rouge et mâles en gris; allaitantes à droite : femelles en noir et mâles en gris) et de l'âge exprimé en jours sur l'axe des abscisses.
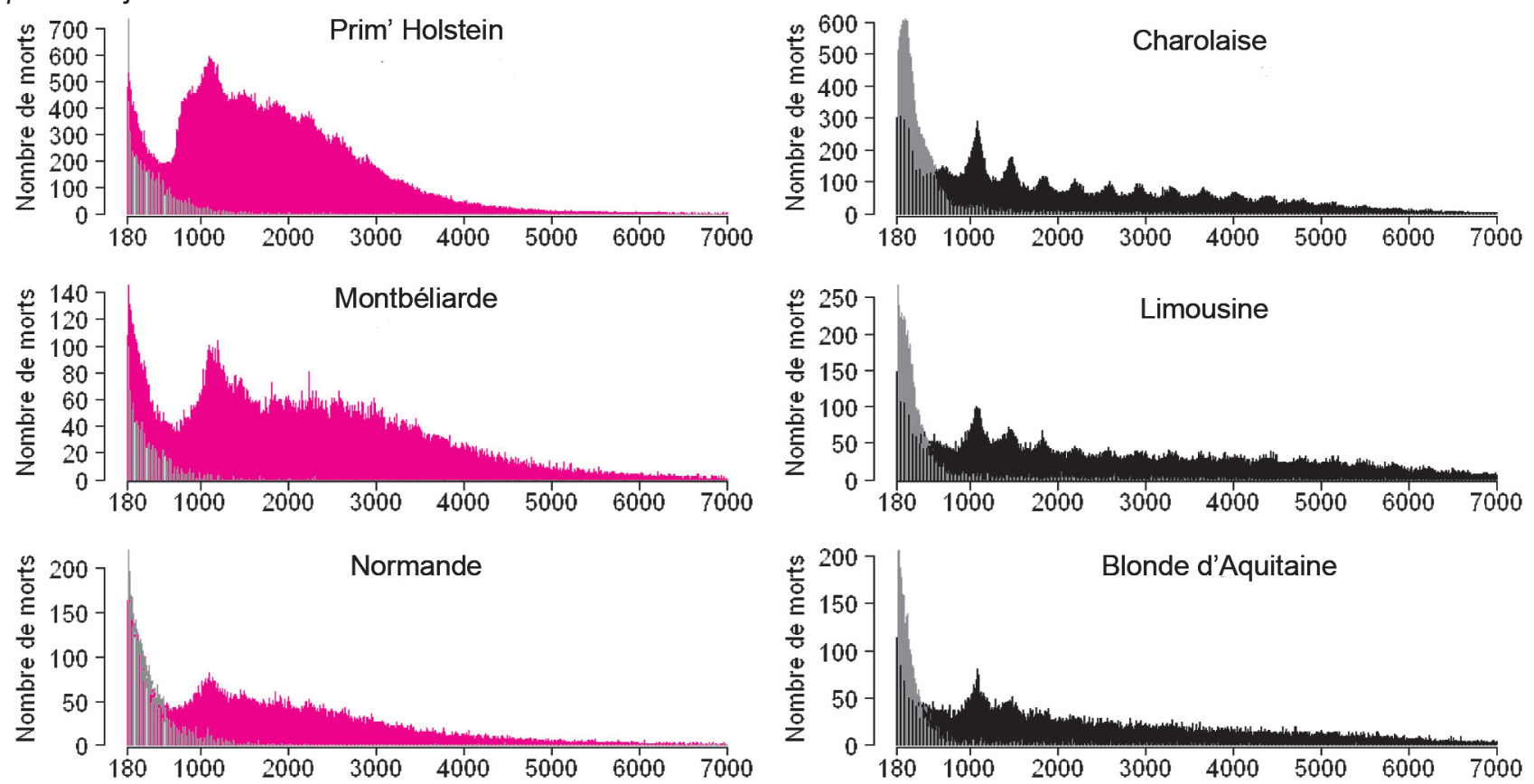

que chez les laitières. On note que plus les vaches laitières ont un profil boucher (Prim'Holstein $<$ Montbéliarde $<$ Normande), plus la distribution du nombre de morts en fonction de l'âge à la mort se rapproche de celle des races allaitantes.

Le nombre de morts par âge décroît après trois ans plus rapidement chez les vaches laitières que chez les allaitantes : parmi les femelles mortes de plus de trois ans, l'âge moyen à la mort était de 6,0 ans chez les laitières et de 8,1 ans chez les allaitantes. Globalement, la vie productive (vêlages et lactation) semble être associée à une mortalité plus importante chez les laitières que chez les allaitantes puisque les taux de mortalité de 2 à 3,5 ans et de 3,5 à 5 ans chez les bovins laitiers sont respectivement 1,7 et 2,0 fois plus élevés que de un à deux ans (tableaux 1 et 2 ), tandis que cette différence est très faible chez les bovins allaitants (rapports respectivement de $0,98$ et 1,07$)$.

Dans la littérature scientifique, la mortalité des adultes est souvent décrite par le risque annuel de mortalité après deux ans. Toutes catégories confondues, le risque annuel de mortalité des animaux de plus de deux ans dans notre population était de $2 \%$ chez les bovins allaitants et 3,5\% de chez les bovins laitiers. Le risque annuel de mortalité chez les bovins laitiers de plus de deux ans varie selon les études de $0,96 \%$ à $6,4 \%$ (Agger et Willeberg 1991, Esslemont et Kossaibati 1997, Faye et Pérochon 1995, Gardner et al 1990, Karuppanan et al 1997, Menzies et al 1995,
Norgaard et al 1999, Stevenson et Lean 1998, Thomsen et al 2004, USDA 2007). Pour les bovins allaitants, les études proposant des valeurs de mortalité pour les animaux de plus de deux ans sont plus rares : risque annuel de $2,4 \%$ en Irlande (Menzies et al 1995), 1,1\% au Canada (Waldner et al 2009), 1,5\% aux Etats-Unis (USDA 2010).

L'écart que nous avons observé chez les bovins adultes entre les deux types de production (cet écart est maximum entre 5 et 10 ans, avec un taux de mortalité 2,4 fois plus élevé chez les laitiers que chez les allaitants) a déjà été rapporté, mais n'est toutefois pas une règle absolue : une étude menée en Irlande a rapporté par exemple des taux de mortalité plus élevés dans le cheptel allaitant que dans le cheptel laitier (Menzies et al 1995).

\section{4 / Evolution temporelle de la mortalité}

\section{a) Saisonnalité}

Le nombre de morts notifiées n'est pas régulier au cours de l'année (figure 5). Une partie de cette variation est liée aux fluctuations de la population (en taille et en âge), elles-mêmes provoquées par la forte saisonnalité des vêlages : le nombre de vêlages présente un pic au mois de mars dans le cheptel allaitant et au mois de septembre dans le cheptel laitier (figure 5).

Mais en parallèle de la population à risque, les taux de mortalité varient eux aussi de manière saisonnière, avec un effet du mois différent selon le sous groupe populationnel considéré (figure 5). L'amplitude des fluctuations saisonnières est plus forte chez les bovins allaitants que chez les laitiers. La plus grande amplitude s'observe chez les veaux allaitants de sept jours à six mois, dont le taux de mortalité est 2,6 fois plus élevé au mois de janvier qu'au mois d'août.

Pour les animaux de plus de sept jours, les taux de mortalité sont au plus haut au cours de l'hiver (pic entre novembre et mars) et au plus bas en été (mai-juin pour les laitiers et juillet-août pour les allaitants). Chez les veaux de moins de sept jours, les taux de mortalité présentent deux pics d'ampleur équivalente : l'un au milieu de l'été (mois de juin-juillet) et l'autre en hiver (mois de décembre).

Les études de la saisonnalité des taux de mortalité des veaux ne sont pas toutes concordantes. Certaines mettent en évidence, conformément à nos résultats, des taux de mortalité plus élevés en hiver (voir Mee et al 2008) pour une revue dans les pays nordiques) et plus faibles au printemps (Bleul 2011). Cette différence est généralement attribuée à l'incidence plus élevée de maladies respiratoires et digestives en hiver (Bleul 2011). D'autres auteurs font par ailleurs état d'un pic de mortalité périnatale au mois de juin comme nous l'avons observé. Dans les pays nordiques, celui-ci est généralement attribué au stress engendré par le retour à la pâture (Auran 1972, Philipsson 1976, Simensen 1982, cités par Mee et al 
Figure 5. Répartition mensuelle des morts et des vêlages notifiés de 2003 à 2009 en France par type de production (laitier en rouge et allaitant en noir), et valeurs des taux de mortalité relatifs mensuels (janvier étant le mois de référence) par classe d'âge.
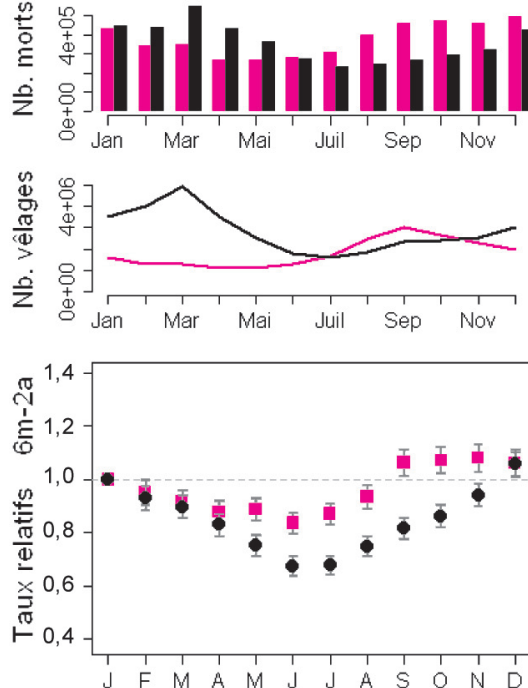

2008). En Irlande où le retour au pré est plus précoce, cette surmortalité des veaux était plutôt attribuée à des troubles métaboliques liés à l'état d'engraissement trop important des vaches gestantes lors de la repousse de printemps, ainsi qu'au manque de surveillance des vêlages tardifs (Mee et al 2008). Comme le suggère cet auteur, nos résultats semblent aussi indiquer que les vêlages hors-saison sont défavorables à la survie des veaux : les taux de mortalité des veaux de moins de sept jours les plus bas étaient observés lors des pics de vêlages (mois de mars chez les allaitants et septembre chez les laitiers). Mais cette observation n'est pas toujours partagée : dans une étude espagnole, le risque de mortalité était par exemple plus faible au début de la saison de vêlage (décembre à février) et augmentait en même temps que le nombre de vêlages (Tarres et al 2005). Les conditions météorologiques pourraient aussi être à l'origine des deux pics de mortalité que nous observons puisque la chaleur estivale et le froid hivernal présenteraient tout deux un risque accru de mortalité des veaux (Martin et al 1975).

Chez les bovins adultes, les études menées font généralement état de taux de mortalité plus faibles en été et plus élevés au printemps (Agger et Willeberg 1991, Faye et Pérochon 1995, Waldner et al 2009). Cette saisonnalité est généralement attribuée à la saison de vêlage, la période peripartum étant une période à risque de mortalité accru pour les vaches. La simultanéité des pics de mortalité chez les adultes et des pics de vêlages que nous avons observée dans chaque type de production (figure 5) conforte cette hypothèse : une augmentation importante des taux de mortalité
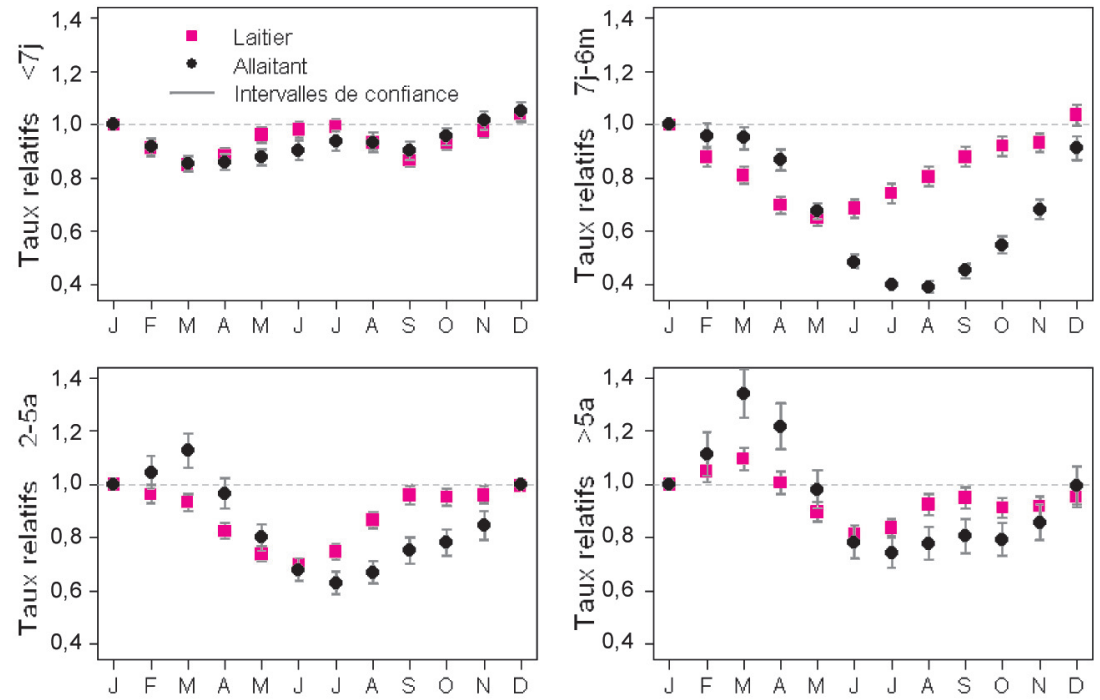

est observée à l'automne chez les bovins laitiers adultes, et au mois de mars chez les bovins allaitants. De même, chez les allaitants, la légère augmentation du nombre de vêlages en septembre, est accompagnée d'une inflexion du taux de mortalité chez les bovins de plus de deux ans.

Toutefois, nous avons aussi constaté que le taux de mortalité des vaches laitières ne décroissait pas après le pic de vêlage automnal, voire augmentait en fin d'hiver, à une période à laquelle le nombre de vêlages laitiers est plutôt décroissant (figure 5). D'autres facteurs, notamment météorologiques (Crescio et al 2010, Stull et al 2008) ou zootechniques (inadaptation du logement hivernal, pression d'infection augmentée en stabulation, troubles métaboliques à la mise à l'herbe...), semblent donc être également impliqués dans la saisonnalité des taux de mortalité des bovins adultes.

\section{b) Tendances de 2003 à 2009}

Les modèles que nous avons ajustés sur les séries chronologiques de mortalité ont identifié dans chaque sous-groupe populationnel une augmentation significative du taux de mortalité au cours de la période 2003-2009 (figure 6). La plus forte augmentation a été observée chez les bovins laitiers de six mois à deux ans, pour qui le taux de mortalité a augmenté en moyenne de $4,3 \%$ (augmentation en valeur relative) par an entre 2003 et 2009.

Pour les veaux de moins de sept jours, l'augmentation annuelle moyenne du taux de mortalité était plus forte chez les bovins allaitants $(2,0 \%)$ que chez les bovins laitiers $(1,5 \%)$. Pour toutes les autres catégories d'âge (de 7 jours à 6 mois, 6 mois à 2 ans, 2 à 5 ans et plus de 5 ans), l'augmentation était plus forte chez les bovins laitiers (respectivement $2,1 \%, 4,3 \%, 2,0 \%, 4,0 \%$ ) que chez les bovins allaitants (respectivement $1,1 \%$, $2,0 \%, 1,0 \%, 2,0 \%)$.

Des modèles similaires ont été ajustés sur la période 2003-2006, en excluant les années 2007-2009, afin d'évaluer l'impact de l'épizootie de Fièvre Catarrhale Ovine (FCO) sur la valeur et la significativité de ces tendances. Sur cette période restreinte, seuls les bovins allaitants de moins de six mois ainsi que les bovins laitiers de moins de sept jours et ceux de plus de cinq ans présentaient encore des tendances croissantes significatives. De 2003 à 2006, les tendances des taux de mortalité des bovins allaitants de plus de six mois étaient en revanche significativement décroissantes, tandis que les taux de mortalité des bovins laitiers des catégories de sept jours à cinq ans ne présentaient pas de tendance significative. Ces résultats suggèrent que les excès de mortalité survenus en 2007 et 2008, alors que l'épizootie de FCO se propageait en France, ont pu influencer les tendances estimées sur la période entière.

De nombreuses études, menées au Danemark (Hansen et al 2004), en Suisse (Bleul 2011), en Irlande (Mee et al 2008), en Suède (Steinbock et al 2003) ou en Amérique du Nord (Meyer et al 2001) rapportent une augmentation de la mortalité périnatale au fil des années, particulièrement dans le cheptel Holstein. D'autres études font aussi état d'une augmentation des taux de mortalité chez les bovins adultes : le risque de mortalité des vaches laitières au Danemark est ainsi passé de $2 \%$ en 
Figure 6. Séries des taux hebdomadaires de mortalité selon le type de production (laitier en rouge et allaitant en noir) et la catégorie d'âge (les lignes en pointillé correspondent aux tendances ajustées par les modèles).
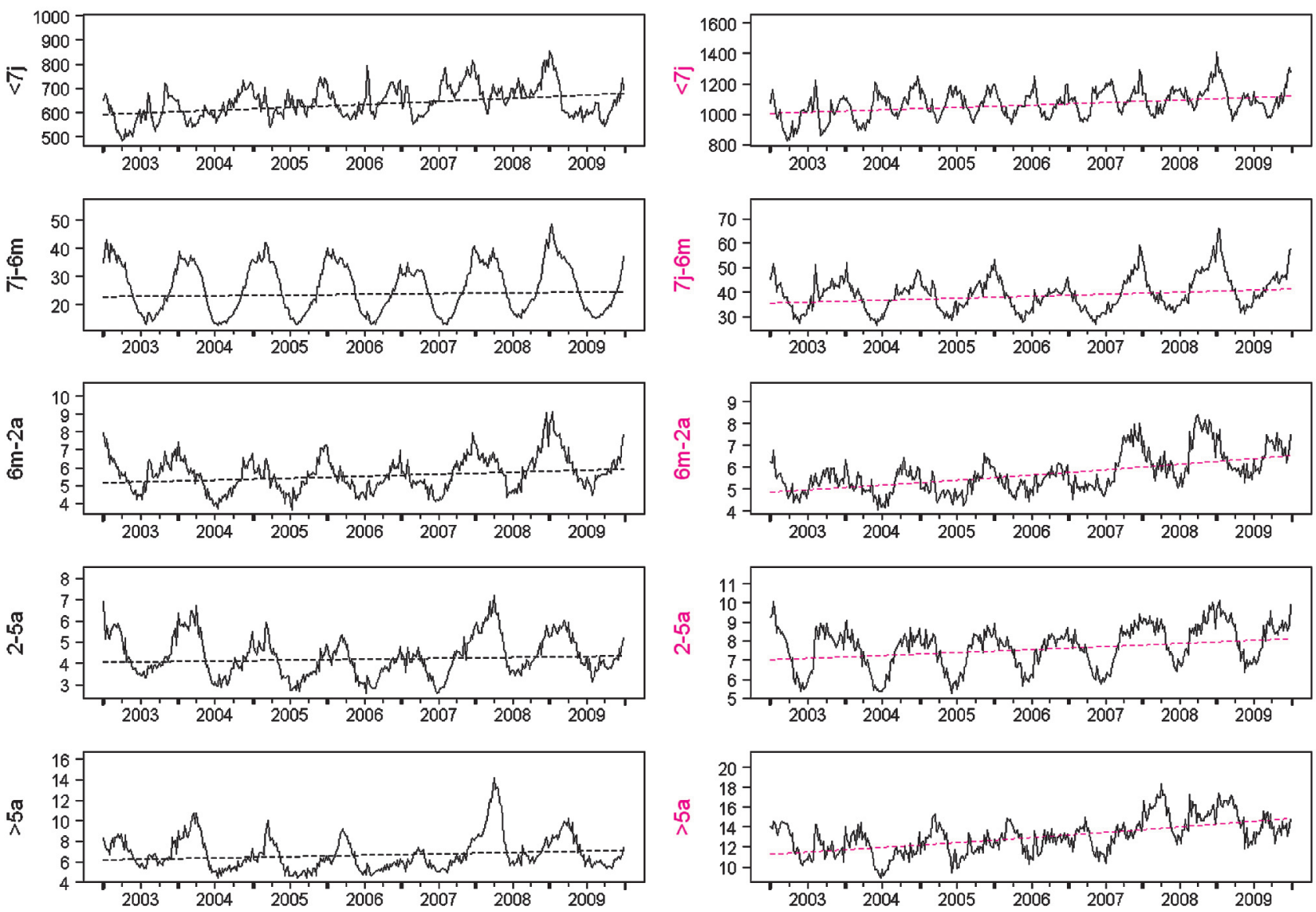

1990 à 3,5\% en 1999 (Thomsen et al 2004) tandis qu'aux Etats-Unis la proportion estimée de vaches mourant chaque année dans le pays est passée de $3,8 \%$ en 1996 à $4,8 \%$ en 2002 puis $5,7 \%$ en 2007 (USDA 2007).

Si les études menées ne permettent pas d'identifier formellement les causes de cette augmentation tendancielle de la mortalité observée dans différents pays, certaines hypothèses ont toutefois été avancées. Aux Etats-Unis, certains auteurs pensent que l'augmentation de la mortalité des bovins laitiers adultes résulte à la fois d'une augmentation de problèmes sanitaires, (qui s'exprime par l'augmentation du nombre de vaches couchées, de problèmes respiratoires, et l'usage croissant d'antibiotiques pour traiter des vaches malades) et d'une évolution des conduites d'élevage (McConnel et al 2008). Le rôle sur la mortalité de l'augmentation de l'incidence de maladies spécifiques, telles que le syndrome hémorragique jéjunal par exemple 1 , semble moins probable car l'augmentation de la mortalité dépasse celle de l'incidence des maladies spécifiques connues. $\mathrm{Au}$
Danemark, les deux hypothèses avancées sont, d'une part l'augmentation du stress physiologique due à l'augmentation de la production et de la consommation de concentrés, et d'autre part la diminution de l'attention portée aux animaux résultant de l'augmentation de la taille des exploitations et de leur mécanisation (Thomsen et al 2004). La proportion croissante de gènes Holstein dans le cheptel laitier est également une hypothèse suggérée pour expliquer l'augmentation des taux de mortalité périnatale (Mee et al 2008).

Au-delà de ces considérations il est par ailleurs probable que l'amélioration de la notification des morts de bovins, ainsi que l'évolution des normes sanitaires (contraignant l'euthanasie d'animaux qui auraient pu autrefois être abattus et consommés) ont pu avoir une influence sur les évolutions observées dans le cheptel français.

\section{Conclusion}

Les valeurs de mortalité bovine proposées dans la littérature scientifique et technique sont extrêmement variables. La mortalité est probablement influencée par de nombreux facteurs spécifiques à chaque population étudiée (pays, systèmes d'élevage, niveau d'intensification...), mais une part de cette variabilité est vraisemblablement attribuable aux différentes méthodes employées pour notifier et quantifier la mortalité. Contrairement à beaucoup de ces études qui étaient basées sur des échantillons relativement réduits et/ou utilisaient des indicateurs approchés (nombre de vaches mortes sur le nombre de vaches ayant vêlé dans l'année, nombre de vaches mortes sur le nombre de vaches recensées au mois de juin...), nous avons réalisé nos estimations à partir d'une base de données très complète (correspondant à l'ensemble de la population bovine française sur sept années, soit 75 millions d'animaux) et en utilisant un dénominateur très précis (nombre de bovin-jours dans chaque sous-groupe populationnel).

L'analyse descriptive des données collectées par la BDNI a ainsi permis d'obtenir des statistiques fiables sur la mortalité bovine en France. Les

\footnotetext{
${ }^{1}$ http://www.extension.org/pages/Adult_Dairy_Cow_Mortality.
} 
effectifs, taux et risques de mortalité calculés offrent une base de référence pouvant être utilisée pour évaluer des situations observées en élevage ou dans une région spécifique.

Les indicateurs estimés révèlent l'importance de la mortalité bovine, particulièrement chez les jeunes animaux. Nous avons constaté une augmentation des taux de mortalité de 2003 à 2009 dans tous les sous-groupes populationnels étudiés. Cette augmentation est probablement en partie due à l'épizootie de FCO qui s'est propagée en France de 2007 à 2008. Toutefois, de nombreuses études étrangères font elles aussi état d'une augmentation des taux de mortalité dans le cheptel bovin, même en l'absence d'épizootie de grande ampleur. Cette évolution défavorable a des conséquences économiques importantes pour les éleveurs et pose la question du bien-être animal. En effet on peut s'étonner que les progrès accomplis en matière de génétique et de zootechnie, ainsi que l'amélioration des matériels agricoles et des connaissances vétérinaires ne soient pas accompagnés d'une diminution de la mortalité dans le cheptel bovin. Une étude plus approfondie de cette augmentation supposée et de ses causes semble nécessaire pour pouvoir proposer d'éventuelles mesures correctrices (amélioration du suivi des vêlages, de la prise de colostrum, des conditions de stabulation hivernale...).

\section{Remerciements}

Les auteurs remercient le Bureau de la Maîtrise d'Ouvrage des Systèmes d'Information de l'Alimentation (BMOSIA) de la Direction Générale de l'Alimentation (DGAL) pour l'accès aux données ainsi qu'André Gauffier pour son implication et son soutien actif au projet.

\section{Références}

Agger J.F., Willeberg P., 1991. Production and mortality in dairy cows from 1960-1990: Time series analysis of ecological data. In: Proceedings of the $6^{\text {th }}$ International Symposium on Veterinary Epidemiology and Economics, August 1991. Ottawa, Canada, 357-360.

Auran T., 1972. Factors affecting the frequency of stillbirths in Norwegian cattle. Acta Agric. Scand., 22, 178-182.

Azzam S.M., Kinder J.E., Nielsen M.K., Werth L.A., Gregory K.E., Cundiff L.V., Koch R.M., 1993. Environmental effects on neonatal mortality of beef calves. J. Anim Sci., 71, 282-290.

Bellows R.A., Patterson D.J., Burfening P.J., Phelps D.A., 1987. Occurrence of neonatal and postnatal mortality in range beef cattle. II. Factors contributing to calf death. Theriogenology, 28, 573-586.

Bleul U., 2011. Risk factors and rates of perinatal and postnatal mortality in cattle in Switzerland. Livest. Sci., 135, 257-264.

Crescio M.I., Forastiere F., Maurella C., Ingravalle F., Ru G., 2010. Heat-related mortality in dairy cattle: A case crossover study. Prev. Vet. Med., 97, 191-197.

Drevenstedt G.L., Crimmins E.M., Vasunilashorn S., Finch C.E., 2008. The rise and fall of excess male infant mortality. Proc. Natl. Acad. Sci. U.S.A., 105, 5016-5021.

Esslemont R.J., Kossaibati M.A., 1997. Culling in 50 dairy herds in England. Vet. Rec., 140, 36-39.

Faye B., Pérochon L., 1995. La mortalité des vaches laitières dans l'enquête écopathologique Bretagne. Vet. Res., 26, 124-131.

Fuerst-Waltl B., Fuerst C., 2010. Mortality in Austrian dual purpose Fleckvieh calves and heifers. Livest. Sci., 132, 80-86

Ganaba R., Bigras-Poulin M., Bélanger D., Couture Y., 1995. Description of cow-calf productivity in Northwestern Quebec and path models for calf mortality and growth. Prev. Vet. Med., 24, 31-42.

Gardner I.A., Hird D.W., Utterback W.W., Danaye-Elmi C., Heron B.R., Christiansen K.H., Sischo W.M., 1990. Mortality, morbidity, case-fatality, and culling rates for california dairy cattle as evaluated by the national animal health monitoring system, 1986-87. Prev. Vet. Med., 8, 157-170.
Goyache F., Gutiérrez J.P., Alvarez I., Fernández I., Royo L.J.,Gómez E., 2003 Genetic analysis of calf survival at different preweaning ages in beef cattle. Livest. Prod. Sci., 83, 13-20.

Gulliksen S.M., Lie K.I., Løken T., Østerås O., 2009. Calf mortality in Norwegian dairy herds. J. Dairy Sci., 92, 2782-2795.

Hansen M., Madsen P., Jensen J., Pedersen J., Christensen L.G., 2003. Genetic parameters of postnatal mortality in Danish holstein calves. J. Dairy Sci., 86, 1807-1817.

Hansen M., Misztal I., Lund M.S., Pedersen J., Christensen L.G., 2004. Undesired phenotypic and genetic trend for stillbirth in Danish Holsteins. J. Dairy Sci., 87, 1477-1486.

Holleville P., Michenot B., 2009. Estimation des pertes par mortalité en élevage. In : Journées Nationales des Groupements Techniques Vétérinaires. SNGTV (Eds). Paris, France, 21-24.

Johanson J.M., Berger P.J., 2003. Birth weight as a predictor of calving ease and perinatal mortality in Holstein cattle. J. Dairy Sci., $86,3745-3755$

Jougla E., 1997. Statistic tests concerning population mortality indicators. Rev. Epidemiol. Sante Publique, 45, 78-84.

Karuppanan P., Thurmond M.C., Gardner I.A., 1997. Survivorship approaches to measuring and comparing cull rates for dairies. Prev. Vet. Med., 30, 171-179.

Lombard J.E., Garry F.B., Tomlinson S.M., Garber L.P., 2007. Impacts of dystocia on health and survival of dairy calves. J. Dairy Sci., 90, 1751-1760.

Losinger W.C., Heinrichs A.J., 1997. Management practices associated with high mortality among preweaned dairy heifers. J. Dairy Res., 64, 1-11.

Martin S.W., Schwabe C.W., Franti C.E., 1975. Dairy calf mortality rate: the association of daily meteorological factors and calf mortality. Can. J. Comp. Med., 39, 377-388.

Martinez M.L., Freeman A.E., Berger P.J., 1983. Factors affecting calf livability for Holsteins. J. Dairy Sci., 66, 2400-2407.

McConnel C.S., Lombard J.E., Wagner B.A., Garry F.B., 2008. Evaluation of factors associated with increased dairy cow mortality on United States dairy operations. J. Dairy Sci., 91, 1423-1432.

Mee J.F., Berry D.P., Cromie A.R., 2008 Prevalence of, and risk factors associated with, perinatal calf mortality in pasture-based Holstein-Friesian cows. Animal, 2, 613-620.

Menzies F.D., Bryson D.G., McCallion T. et Matthews D.I., 1995. A study of mortality among suckler and dairy cows in Northern Ireland in 1992. Vet Rec., 137, 531-536.

Meyer C.L., Berger P.J., Koehler K.J. Thompson J.R., Sattler C.G., 2001. Phenotypic trends in incidence of stillbirth for Holsteins in the United States. J. Dairy Sci., 84, 515-523.

Nix J.M., Spitzer J.C., Grimes L.W., Burns G.L., Plyler B.B., 1998. A retrospective analysis of factors contributing to calf mortality and dystocia in beef cattle. Theriogenology, 49, 15151523 .

Norberg E., 2008. A genetic study of postnatal mortality in Danish Jersey heifer calves. In: $59^{\text {th }}$ Annual meeting of the European Association for Animal Production, 24-27 August 2008, Vilnius, Lithuania.

Norgaard N.H., Lind K.M., Agger J.F., 1999. Cointegration analysis used in a study of dairycow mortality. Prev. Vet. Med., 42, 99-119.

Offredo T., 2010. Mortalité des veaux en élevages allaitants. Terragricoles-de-Bretagne, 227,35 .

Patterson D.J., Bellows R.A., Burfening P.J., Carr J.B., 1987. Occurrence of neonatal and postnatal mortality in range beef cattle. I. Calf loss incidence from birth to weaning, backward and breech presentations and effects of calf loss on subsequent pregnancy rate of dams. Theriogenology, 28, 557-571.

Perrin J.B., Ducrot C., Vinard J.L., Morignat E., Gauffier A., Calavas D., Hendrikx P., 2010. Using the National Cattle Register to estimate the excess mortality during an epidemic: Application to an outbreak of Bluetongue serotype 8. Epidemics, 2, 207-214.

Philipsson J., 1976. Studies on calving difficulty, stillbrith and associated factors in Swedish cattle breeds. II. Effects of non-genetic factors. Acta Agriculturae Scandinavica, 26, $165-174$

Rao M.K., Nagarcenkar R., 1980. Calf mortality in crossbred dairy cattle. Trop. Anim. Health Prod., 12, 137-144. 
Schumann F.J., Townsend H.G., Naylor J.M., 1990. Risk factors for mortality from diarrhea in beef calves in Alberta. Can. J. Vet. Res., 54, 366-372.

Simensen E., 1982. An epidemiological study of calf health and performance in Norwegian dairy herds. II. Factors affecting mortality. Acta Agriculturae Scandinavica, 32, 421-427.

Steinbock L., Nasholm A., Berglund B., Johansson K., Philipsson J., 2003. Genetic effects on stillbirth and calving difficulty in Swedish Holsteins at first and second calving. J. Dairy Sci., 86, 2228-2235.

Stevenson M.A., Lean I.J., 1998. Descriptive epidemiological study on culling and deaths in eight dairy herds. Aust. Vet. J., 76, 482-488.

Stull C.L., Messam L.L.M.V., Collar C.A., Peterson N.G., Castillo A.R., Reed B.A., Andersen K.L., VerBoort W.R., 2008. Precipitation and temperature effects on mortality and lactation parameters of dairy cattle in California. J. Dairy Sci., 91, 4579-4591.
Svensson C., Linder A., Olsson S.O., 2006. Mortality in Swedish dairy calves and replacement heifers. J. Dairy Sci., 89, 4769-4777.

Tarres J., Casellas J., Piedrafita J., 2005. Genetic and environmental factors influencing mortality up to weaning of Bruna dels Pirineus beef calves in mountain areas. A survival analysis. J. Anim. Sci., 83, 543-551.

Thomsen P.T. Kjeldsen A.M., Sorensen J.T. Houe H., 2004. Mortality (including euthanasia) among Danish dairy cows (1990-2001). Prev. Vet. Med., 62, 19-33.

Torsein M., Lindberg A., Sandgren C.H. Waller K.P., Törnquist M., Svensson C., 2011 Risk factors for calf mortality in large Swedish dairy herds. Prev. Vet. Med., sous presse.

USDA, 2007. Dairy 2007, Part I: Reference of Dairy Cattle Health and management Practices in the United States, 2007. United States Department of Agriculture, Animal and Plant Health Inspection Service, 128.

USDA, 2010. Mortality of calves and cattle on U.S. beef cow-calf operations. APHIS Info sheet, United States Department of
Agriculture, Animal and Plant Health Inspection Service.

Virtala A.M., Mechor G.D., Grohn Y.T., Erb H.N., 1996. Morbidity from non respiratory diseases and mortality in dairy heifers during the first three months of life. J. Am. Vet. Med. Assoc., 208, 2043-2046.

Waldner C.L., Kennedy R.I., Rosengren L., Clark E.G., 2009. A field study of culling and mortality in beef cows from western Canada. Can. Vet. J., 50, 491-499.

Waldner C.L., Kennedy R.I., Rosengren L.B., Pollock C.M., Clark E.T., 2010. Gross postmortem and histologic examination findings from abortion losses and calf mortalities in western Canadian beef herds. Can. Vet. J., 51, 1227-1238.

Wittum T.E., Salman M.D., Odde K.G., Mortimer R.G., King M.E., 1993. Causes and costs of calf mortality in Colorado beef herds participating in the National Animal Health Monitoring System. J. Am. Vet. Med. Assoc., 203, 232-236.

\section{Résumé}

La mortalité bovine est un problème important, tant au plan économique que du point de vue du bien-être animal. Nous avons analysé les données enregistrées dans la base de données nationale d'identification de 2003 à 2009 , décrivant la carrière d'environ $\mathbf{7 5}$ millions de bovins, afin de fournir des statistiques fiables de la mortalité bovine en France. Les résultats de cette étude constituent un référentiel pouvant être utilisé pour évaluer des situations observées en élevage.

En moyenne sur cette période, le risque de mortalité périnatale des mâles et des femelles atteignait respectivement $7,9 \%$ et $6,0 \%$ dans le cheptel laitier, et $4,7 \%$ et $3,2 \%$ dans le cheptel allaitant. Le risque de mortalité annuel moyen des animaux de plus de deux ans était de $3,5 \%$ chez les laitiers et de $\mathbf{2 , 0} \%$ chez les allaitants. La mortalité des bovins laitiers était significativement plus élevée que celle des allaitants pour les dix classes d'âge étudiées, sauf celle de un à deux ans. La modélisation des données hebdomadaires a permis de décrire la saisonnalité des taux de mortalité, qui était différente selon le sous-groupe populationnel considéré. Les taux de mortalité des veaux de moins de sept jours présentaient notamment deux pics d'ampleur équivalente (en décembre et juillet), tandis que les autres classes d'âge présentaient un pic principal en hiver, visiblement associé chez les adultes au pic de vêlage. Les résultats de cette modélisation ont par ailleurs révélé une augmentation significative des taux de mortalité de 2003 à 2009 , probablement en partie due à l'épizootie de fièvre catarrhale ovine qui s'est propagée en France en 2007-2008. L'augmentation des taux de mortalité bovine a déjà été constatée dans de nombreux pays, suggérant la nécessité de mettre en place des mesures correctrices.

\section{Abstract}

\section{Analysis of cattle mortality in France, 2003-2009}

Cattle mortality represents both an economical and an animal welfare issue. We analyzed the data recorded in the National Cattle Register from 2003 to 2009, gathering data about 75 million cattle, to provide reliable statistics of cattle mortality in France.

On average over this period, the perinatal mortality risk in males and females reached respectively $7.9 \%$ and $6.0 \%$ in dairy cattle, and $4.7 \%$ and $3.2 \%$ in beef cattle. The average annual mortality risk of animals over two years was $3.5 \%$ for dairy and $2.0 \%$ for beef cattle. Mortality of dairy cattle was significantly higher than that of beef cattle for the ten age groups studied, except for those of one to two years. Weekly data were modeled to describe the seasonality of mortality, which was different among the different sub-population groups. The mortality rate of calves less than seven days old showed two peaks of equal magnitude (in December and July), while other age groups showed a major peak in the winter, apparently associated in adults to the peak of calving. The modeling results of the model also revealed a significant increase in death rates from 2003 to 2009 , probably partly due to the blue tongue epizootic that spread in France from 2007 to 2008. Rising rates of cattle mortality have already been observed in many countries, suggesting the need to implement corrective measures.

PERRIN J.-B., DUCROT C., VINARD J.-L., HENDRIKX P., CALAVAS D., 2011. Analyse de la mortalité bovine en France de 2003 à 2009. INRA Prod. Anim., 24, 235-244. 